\title{
Florence robotic intracorporeal neobladder (FloRIN): a new reconfiguration strategy developed following the IDEAL guidelines
}

\author{
Andrea Minervini*(D, Davide Vanacore*, Gianni Vittori*, Martina Milanesi*, \\ Agostino Tuccio*, Giampaolo Siena*, Riccardo Campi*, Andrea Mari*, \\ Andrea Gavazzi ${ }^{\dagger}$ and Marco Carini*
}

${ }^{*}$ Department of Urology, University of Florence, Florence, Italy, and 'Azienda Sanitaria di Firenze (ASF), Florence, Italy

\section{Objective}

To describe our step-by-step technique for robotic intracorporeal neobladder configuration, including the stages of conception, development and exploration of this surgical innovation, according to the Idea, Development, Exploration, Assessment, Long-term follow-up (IDEAL) Collaboration guidelines.

\section{Patients and Methods}

The Florence robotic intracorporeal neobladder (FloRIN) was performed employing the following main surgical steps: isolation of $50 \mathrm{~cm}$ of ileum; bowel anastomosis; urethro-ileal anastomosis creating an asymmetrical ' $U$ '-shape $(30 \mathrm{~cm}$ distally and $20 \mathrm{~cm}$ proximally to anastomosis), ileum detubularisation; posterior wall reconfiguration as an ' $\mathrm{L}$ '; bladder neck reconstruction; anterior folding of the posterior plate to reach the 12 o'clock position; uretero-enteral 'orthotopic' bilateral anastomosis. The conception and development of the FloRIN followed the IDEAL guidelines recommended stages: Phase 1 (simulation) involved the neobladder robotic configuration using silicone models. Phase 2a (development) aimed to reproduce the configuration in an open fashion in one patient, and then in the first three robotic procedures. Phase $2 \mathrm{~b}$ (exploration) consisted of the technique standardisation in 15 consecutive robotic approaches. Phase $2 \mathrm{a}$ and $2 \mathrm{~b}$ included urodynamics and imaging assessment of the patients treated.

\section{Results}

From February 2016 to September 2017 FloRIN was performed in 18 patients. Comparing the first three (Phase $2 \mathrm{a}$ ) with the subsequent 15 patients (Phase $2 \mathrm{~b}$ ), the median (interquartile range $[\mathrm{IQR}]$ ) reconstruction operating time was 260 (220-340) vs 160 (150-210) min, respectively.

Postoperative surgical complications occurred in four of the 18 patients $(22.1 \%)$, including one surgical Clavien-Dindo Grade III and three Grade I, postoperative medical ClavienDindo Grade II complications occurred in three (16.7\%) patients. On urodynamic examination (available in nine [50\%] patients) the median (IQR) reservoir cystometric capacity, compliance, and post-void residual were 240 (220267) $\mathrm{mL}, 18(12.5-19.8) \mathrm{mL} / \mathrm{cmH}_{2} \mathrm{O}$, and 0 (0-50) $\mathrm{mL}$, respectively. Ultrasonography showed no Grade $\geq 2$ vesicoureteric reflux.

\section{Conclusion}

We describe the FloRIN configuration, showing its technical feasibility with acceptable time efficiency. The first cases studied had good reservoir capacity, low pressure with no reflux, and complete voiding.

\section{Keywords}

robot-assisted radical cystectomy, orthotopic ileal neobladder, intracorporeal, urinary diversion, IDEAL guidelines

\section{Introduction}

Robot-assisted radical cystectomy (RARC) is a widespread minimally invasive technique for the treatment of muscleinvasive and organ-limited bladder cancer in selected patients. The intracorporeal neobladder (ICNB) remains a complicated and technically demanding and time-consuming surgical step, thus many centres adopt a hybrid approach that comprises a

robotic demolition phase and an open reconstructive step with the urethro-ileal anastomosis made robotically after re-docking [1]. Patients who undergo extracorporeal neobladder seem to have a comparable overall postoperative surgical complication rate, but higher gastrointestinal complications [1]. Several revisions of standard open neobladder configurations have been proposed for the robotic approach, but the perfect ICNB has not been unanimously identified $[2,3]$. The optimum 
reservoir should have adequate capacity for storage, low pressure to avoid any damage to the kidneys, high compliance to help in continence, and should allow voluntary emptying without residual urine. In order to translate such surgical principles and to keep the neobladder formation technique as simple as possible, we designed an innovative model of robotic ICNB based on a double bowel reconfiguration and 'orthotopic' uretero-ileal anastomoses on each side of the reservoir's neo-trigone, which allows for faster reconstruction and facilitates the endoscopic management of the ureters in case of any future pathologies of the upper urinary tract (e.g. stenosis, stones, upper tract urothelial cell carcinoma). However, further studies are needed to test this hypothesis. The Balliol Collaboration recommended a series of guidelines, the Idea, Development, Exploration, Assessment, Long-term follow-up (IDEAL) Collaboration guidelines, which allow surgical innovation whilst minimising complications, through a framework that includes the Idea (phase 1), the Development (phase 2a), the Exploration (phase 2b) and the Assessment (phase 3) phases [4]. According to the IDEAL guidelines, we adhered to the following steps: Phase 1 (idea) focused on explanation and description by schematics, and robotic configuration of the neobladder in silicone models. Phase 2a (development) focused on technical details and surgical feasibility, reproducing the personal configuration in an open fashion in one patient and then in the first three robotic procedures. Phase $2 \mathrm{~b}$ (exploration) included robotic technique standardisation in 15 consecutive patients (technique now more stable and replicated by others). Postoperative urodynamic and imaging assessment were included in phase $2 \mathrm{a}$ and $2 \mathrm{~b}$. In the accompanying video we describe the FloRIN.

\section{Surgical Technique}

All the reconstructive phases were performed by a single surgeon (A.M.) experienced in robotic reconstructive surgery, with $>1000$ open and $>1000$ robotic procedures.

\section{Demolition Phase}

Robot-assisted radical cystectomy and extended lymphadenectomy were performed using the Da Vinci ${ }^{\circledR}$ robot (Intuitive Surgical, Sunnyvale, CA, USA) with a four-arm configuration. The patient was placed in a $30^{\circ}$ Trendelenburg with a standard six-port transperitoneal approach [5] and then reduced to $20^{\circ}$ once the extirpative part was completed for orthotopic ileal neobladder formation to facilitate bowel handling and urethro-ileal anastomosis.

\section{Bowel Isolation and Continuity Restoration}

The caecum is identified and the first stay suture is placed on the ileum $20 \mathrm{~cm}$ proximally to the ileocaecal valve. Then a
$50 \mathrm{~cm}$ segment of ileum is chosen according to its capacity to reach the urethra with the least tension, and a second mark suture is placed.

The ileum is then sectioned at the marked points with endoGIA $60 \mathrm{~mm}$ Echelon Powered Endopath ${ }^{\circledR}$ Stapler (Ethicon Inc., Cincinnati, OH, USA), and the intestinal continuity is restored with an intracorporeal side-to-side anastomosis with one longitudinal and one horizontal fire.

\section{Urethro-ileal Anastomosis}

The urethro-ileal anastomosis is made on a 20-F Foley catheter en bloc with the posterior reconstruction of the rhabdomyosphincter as previously described [6], taking care to create an asymmetrical ' $U$ '-shape with the segment of bowel, $30 \mathrm{~cm}$ distally and $20 \mathrm{~cm}$ proximally to the anastomosis. For this purpose, a 3-0 STRATAFIX ${ }^{\mathrm{TM}}$ suture $(16 \times 16 \mathrm{~cm}$, two $\times$ RB-1 taper, double armed; Ethicon Inc.) is used.

\section{The FloRIN Reconfiguration}

The main phases of FloRIN, illustrated simultaneously in schematics in the silicone model and in vivo during robotic ICNB, are shown in Fig. 1. The ileum segment is entirely detubularised and the posterior plane is reconfigured as an ' $\mathrm{L}$ ', in order to widen the posterior support of the neobladder, creating a neo-trigone. After the completion of the posterior plate, the neobladder neck is reconstructed suturing a tract of $2-5 \mathrm{~cm}$ longitudinally from the 12 o'clock position of the anastomosis, upwards. Then, the posterior plate is folded anteriorly, roughly $5 \mathrm{~cm}$ right from the proximal edge of the posterior closure to create two symmetrical segments. It should be noted that the length of the neobladder neck is decided according to the capacity of the posterior plate to reach the 12 o'clock position of the anastomosis, when folded. The easier the folding is, the shorter the neobladder neck should be.

The ureteric ends are adequately spatulated, and then 'orthotopically' re-implanted on the lateral side of each anterior segment. Ureteric re-implantation is done with no anti-reflux mechanisms, on mono J ureteric catheters, with 4-0 poliglecaprone 25 absorbable sutures (Monocryl; Ethicon Inc.), using two semi-continuous or interrupted sutures.

The anterior plate is finally closed with an inverted ' $\mathrm{V}$ 'shaped suture and both mono J stents are passed through the closing of the two anterior inverted ' $\mathrm{V}$ '-shape plates. All the reconfigurations are done using V-Loc ${ }^{\mathrm{TM}}$ 3-0 monofilament barbed sutures (Covidien Ltd, Dublin, Republic of Ireland). The neobladder is filled with $100 \mathrm{~mL}$ saline to confirm watertightness. 
Fig. 1 Surgical steps of the FloRIN configuration, simultaneously shown (horizontal row) in schematics, in silicone models robotically shaped, and in vivo during ICNB. (A) Detubularised asymmetrical 'U'-shape segment of bowel, $30 \mathrm{~cm}$ distally and $20 \mathrm{~cm}$ proximally to the anastomosis. The posterior plane is reconfigured as an ' $L$ ', by suturing the span of the arms of the ' $U$ ' aligned in parallel, and by placing the extending portion of the loop distally to the right, forming the short arm of the 'L'. (B) The neobladder neck is constructed by suturing a tract of $2-5 \mathrm{~cm}$ longitudinally from the 12 o'clock position of the anastomosis, upwards. (C) The posterior plate is folded anteriorly, distal to proximally, roughly $5 \mathrm{~cm}$ right from the proximal edge of the posterior closure with the goal of creating two symmetrical segments. (D) The ureters are re-implanted, on mono J ureteric catheters, orthotopically and directly with no anti-reflux mechanisms on the lateral side of each anterior segment. The anterior plate is finally closed with an inverted ' $\mathrm{V}$ '-shaped suture and both mono $\mathrm{J}$ stents are passed through the closing of the two sutures.

A

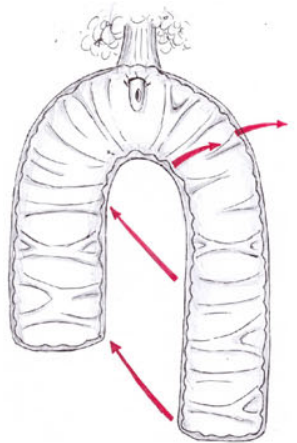

B

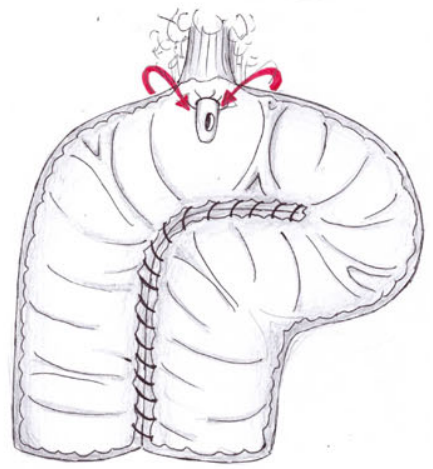

$\mathrm{X}$

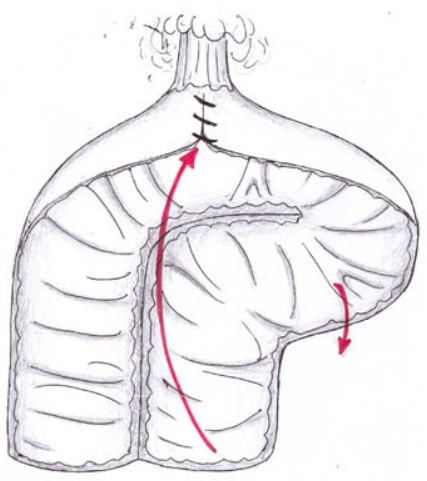

$\Delta$

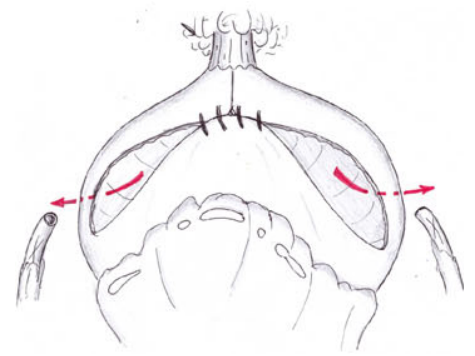

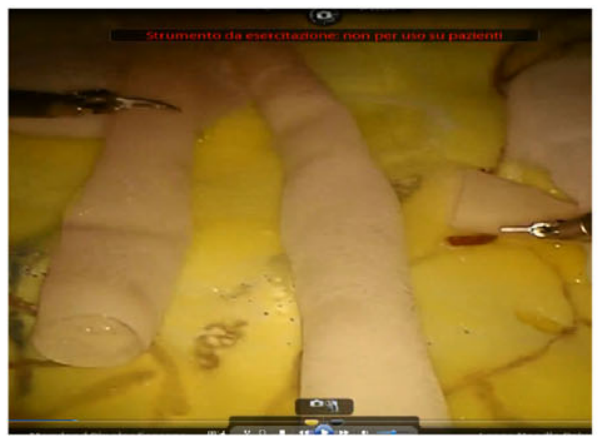
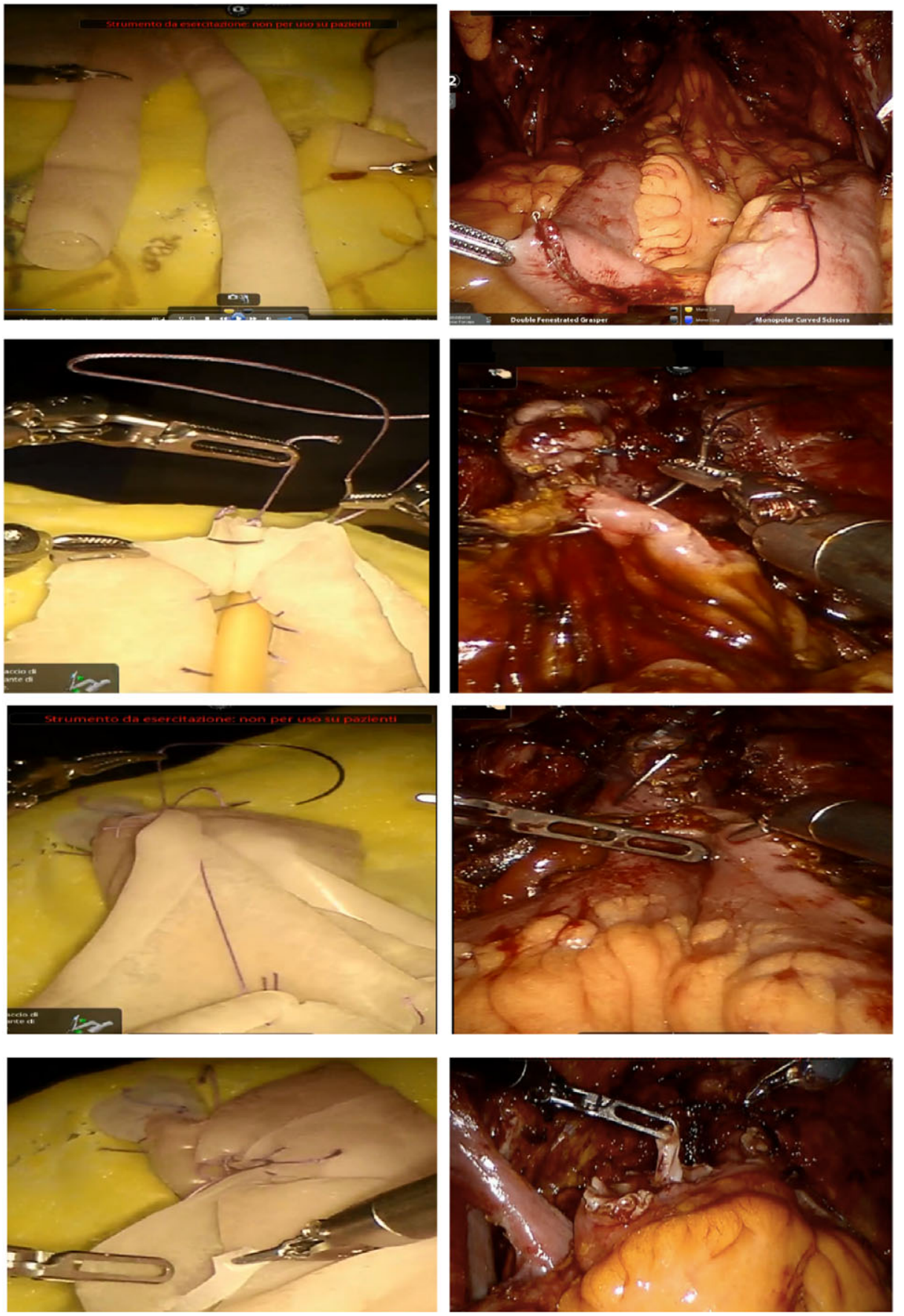


\section{Postoperative Care}

All patients were counselled preoperatively for an Enhanced Recovery After Surgery (ERAS) protocol. As such, the nasogastric tube was removed after surgery. From the first postoperative day (POD) patients were fed, started physiotherapy and mobilised. The abdominal drain was usually removed on the first POD and the patients were discharged on the seventh POD. In outpatients, ureteric catheters were removed between the 10th and 12th POD, and the urethral catheter on the 21st POD.

\section{Preliminary Results}

Overall, the FloRIN was performed in 18 patients (16 were males). The median (range) age was 66 (48-77) years. Clinical and epidemiological characteristics are summarised in Table S1. The median overall (from first incision to skin closure) operating and console (operating time at the robotic console) time was 450 and $410 \mathrm{~min}$, respectively. In the first three (Phase 2a) and in the subsequent 15 patients (Phase $2 \mathrm{~b})$, the median (interquartile range [IQR]) reconstruction operating time was $260(220-340)$ and $160(150-210) \mathrm{min}$, respectively. Overall, 14 (80\%) patients adhered to the ERAS pathway, whilst three (20\%) patients were excluded from the protocol for nausea not responding to medication and were treated with standard perioperative care. No intraoperative complications were recorded. Postoperative surgical complications occurred in four of the 18 patients (22.1\%) including: one surgical Clavien-Dindo Grade III and three Grade I, postoperative medical Clavien-Dindo Grade II complications occurred in three of the 18 patients (16.7\%). One (5.5\%) patient had bilateral ureteric stenosis with hydronephrosis and lymphocoele, and underwent bilateral nephrostomy placement (Clavien-Dindo Grade IIIa), drain placement (Clavien-Dindo Grade IIIa), and consequently bilateral ureteroneocystostomy (Clavien-Dindo Grade IIIb). Three (16.7\%) patients had wound infections. Two (11.1\%) patients with persistent postoperative fever $\left(>38^{\circ} \mathrm{C}\right)$ were treated with i.v. antipyretics, antibiotics and rehydration (Clavien-Dindo Grade II). One (5.5\%) patient had postoperative pneumonia treated with antibiotics (ClavienDindo Grade II) and bowel sub-obstruction treated with oral administration of diatrizoate sodium solution.

Overall, 14 (77.8\%) patients were day-time continent at the last follow-up. The four incontinent patients had a median (range) follow-up of 3 (2-6) months. Furthermore, 12 (66.7\%) patients reported night-time continence awakening twice a night to void. No patient used postoperative clean intermittent catheterisation. Urodynamic evaluation was performed 6 months after surgery and available for nine of the 18 patients. The median (IQR) reservoir maximum cystometric capacity, compliance, and post-void residual were
$240(220-267) \mathrm{mL}, 18(12.5-19.8) \mathrm{mL} / \mathrm{cmH}_{2} \mathrm{O}$, and $0(0-$ 50) $\mathrm{mL}$, respectively. Ultrasonography showed complete voiding in 13 of the 18 patients, with no Grade $\geq 2$ VUR. Functional outcomes and voiding patterns are summarised in Table S2.

\section{Summary}

The FloRIN configuration is technically feasible with acceptable time efficiency. The first cases studied showed good reservoir capacity, low pressure with no reflux, and complete voiding. The IDEAL approach allowed us to minimise complications without the help of a mentor. Further prospective clinical studies with larger patient cohorts are necessary to validate our surgical technique and to assess the long-term postoperative complications, as most occur within the first 2 years [1,7], to confirm the long-term functional outcomes, the oncological validity, and to define the patient selection criteria for this surgical procedure.

\section{Acknowledgments}

We would like to thank Dr Chiara Cini for the video narration.

\section{Conflict of interests}

None.

\section{References}

1 Hussein AA, Ahmed YE, Kozlowski JD et al. Robot-assisted approach to ' $W$ '-configuration urinary diversion: a step-by-step technique. BJU Int 2017; 120: 152-7

2 Chopra S, de Castro Abreu AL, Berger AK et al. Evolution of robotassisted orthotopic ileal neobladder formation: a step-by-step update to the University of Southern California (USC) technique. BJU Int 2017; 119: 185-91

3 Hautmann S, Chun KH, Currlin E, Braun P, Huland H, Juenemann KP. Refluxing chimney versus nonrefluxing LeDuc ureteroileal anastomosis for orthotopic ileal neobladder: a comparative analysis for patients with bladder cancer. J Urol 2006; 175: 1389-93

4 Barkun JS, Aronson JK, Feldman LS, Maddern GJ, Strasberg SM, for the Balliol Collaboration. Evaluation and stages of surgical innovation. Lancet 2009; 374: 1089-96

5 Chopra S, Metcalfe C, de Castro Abreu AL et al. Port placement and docking for robotic surgery: the University of Southern California Approach. J Endourol 2015; 29: 868-72

6 Wiklund NP, Poulakis V. Robotic neobladder. BJU Int 2011; 107: 1514-37

7 Hussein AA, Hashmi Z, Dibaj S et al. Reoperations following robotassisted radical cystectomy: a decade of experience. J Urol 2016; 195: $1368-76$

Correspondence: Andrea Minervini, Associate Professor of Urology, Department of Urology, Careggi Hospital, Padiglione San Luca Nuovo, Largo Brambilla, 50139 Florence, Italy.

e-mail: andreamine@libero.it 
Abbreviations: ERAS, enhanced recovery after surgery; ICNB, intracorporeal neobladder; IDEAL, Idea, development, exploration, assessment, long-term follow-up; IQR, interquartile range; POD, postoperative day; RARC, robotassisted radical cystectomy.

\section{Supporting Information}

Additional Supporting Information may be found in the online version of this article:
Table S1 Preoperative characteristics of the 18 patients who underwent FloRIN.

Table S2 Functional outcomes and voiding frequencies of the 18 patients who underwent FloRIN.

Video S1 Step-by-step technique for robotic intracorporeal neobladder configuration. 